\title{
Drosophila melanogaster as a model host to study arbovirus-vector
}

\section{interaction}

3

4 Mandar S. Paingankar ${ }^{1,2}$, Mangesh D. Gokhale ${ }^{3}$, Deepti D. Deobagkar ${ }^{1,4}$ and Dileep N.

5 Deobagkar ${ }^{1} *$

6

1. Molecular Biology Research Laboratory, Centre of Advanced Studies, Department of Zoology, Savitribai Phule Pune University, Pune, India, 411007.

2. Department of Zoology, Government Science College, Chamorshi Road, Gadchiroli, Maharashtra, India, 442605.

3. Department of Medical Entomology and Zoology, National Institute of Virology, Pune, India. University, Pune, India, 411007. 


\section{ABSTRACT}

Arboviruses cause the most devastating diseases in humans and animals worldwide. Several hundred arbovirus are transmitted by mosquitoes, sand flies or ticks and are responsible for more than million deaths annually. Development of a model system is essential to extrapolate the molecular events occurring during infection in the human and mosquito host. Virus overlay protein binding assay (VOPBA) combined with MALDI TOF/TOF MS revealed that Dengue-2 virus (DENV-2) exploits similar protein molecules in Drosophila melanogaster and Aedes aegypti for its infection. Furthermore, the virus susceptibility studies revealed that DENV-2 could propagate in $D$. melanogaster, and DENV-2 produced in fruit fly is equally infectious to D. melanogaster and Ae. aegypti. Additionally, real time PCR analysis revealed that RNAi coupled with JAK-STAT and Toll pathway constitutes an effector mechanism to control the DENV-2 infection in flies. These observations point out that D. melanogaster harbors all necessary machineries to support the growth of arboviruses. With the availability of well-established techniques for genetic and developmental manipulations, D. melanogaster, offers itself as the potential model system for the study of arbovirus-vector interactions.

33

34 Keywords: Arbovirus-vector interactions, house keeping molecules, insect model system, RNAi, 35 virus overlay protein binding assay 


\section{INTRODUCTION}

The molecular and biochemical basis of the invertebrate host specificity to arboviruses is interesting but comparatively less understood phenomenon. Most of the virus/parasite interacting proteins are house keeping in nature ${ }^{1-5}$ (also see Supplementary Information Table S1). For example, actin, Heat shock proteins, prohibitin and tubulin are reported as arbovirus interacting proteins in Aedes cells $^{2,3,5-7}$. These polypeptides are present ubiquitously in other insects also. As parasites/viruses are dependent on their symbionts for reproduction, we might expect that it would be advantageous for parasites/viruses to have broad host ranges and an abundance of potential vectors. Susceptibility, early multiplication and production of virulent Plasmodium gallinaceum in non-vector non hematophagous insect Drosophila melanogaster support this hypothesis ${ }^{8}$. Arboviruses infect more than 100 million people worldwide every year. The cellular mechanism for transmission and the complex molecular interplay between arboviruses and their vectors are not well characterized. It has hampered development of novel strategies for disease intervention and control. Genomic sequences are available for various diseases causing vectors in literature and Genomic databases ${ }^{8}$. Molecular mechanisms associated with virus propagation in mosquito have been studied using genomics approach in Ae. aegypti and Anopheles mosquitoes ${ }^{10-12}$. In these two species, the availability of the whole genome sequences helped in identification of possible molecular mechanisms involved in virus infections. However, in many other insect vectors such as ticks, sand flies, fleas and other members of Culicidae family, limited specific proteomics information limit the in depth understanding of vector parasite interaction in these species. Considering the sequence and function similarities in the receptor polypeptide components, house keeping proteins, innate immune system genes and other regulatory gene sequences in the eukaryotes, it could be feasible to establish a test system for the study invertebrate parasite/virus interactions.

The Drosophila model system has been explored to study a variety of human infections and diseases $^{12-18}$. Furthermore, the utility of D. melanogaster as a host model system for understanding cellular interactions of various human pathogens has been well-established ${ }^{8,19-28}$. However, $D$. 
melanogaster underutilized in arbovirus studies and holds great potential in the understanding mechanisms involved in arbovirus pathogenesis. Therefore, as a step towards understanding the molecular mechanisms involved in arbovirus-vector interactions, the present work seeks to develop a Drosophila model of dengue infection that better reflects the molecular events in the human and mosquito infections.

In the current study, we report that $D$. melanogaster can serve as a useful model system for the growth and the propagation of DENV-2. The virus produced in D. melanogaster can also infect the Ae. aegypti similar to the virus grown in mosquito. We report that the infectivity and multiplication of the virus grown in D. melanogaster and Ae. aegypti is comparable. Drosophila model system has biosafety advantage over Ae.aegypti, as Drosophila do not feed on blood and never transmit any infectious diseases though bite. Therefore, the application of this model system thus could also be extended to the other arboviral infection analysis.

\section{MATERIAL AND METHODS}

\section{Ethics statement}

Rules laid by Institutional Animal Ethics Committee (IAEC) affiliated with National Institute of Virology (NIV), Pune, India were followed for handling of animals. These experiments were carried out in a biosafety level-2 facility of the NIV. All animal experiments were approved by the IAEC and experiments were performed as per the guidelines laid by the Committee for the Purpose of Control and Supervision of Experiment on Animals (CPCSEA), India.

\section{Drosophila flies and Ae. aegypti mosquito}

Oregon K stocks of D. melanogaster were grown on standard cornmeal-agar medium at $24^{\circ} \mathrm{C}$. Ae. aegypti mosquitoes were reared in laboratory conditions at $28 \pm 1{ }^{\circ} \mathrm{C}, 70 \pm 5 \%$ relative humidity (RH) and light: dark (LD) 12:12 h. Adult mosquitoes and flies, 3-6 days of age were used in the infection experiments.

\section{Virus stock preparation}


Virus stock was prepared by inoculating TR1751 strain of DENV-2 in mice by following the procedure described earlier ${ }^{29}$. Virus titer of randomly picked vial was determined by plaque assay $\left(8.23 \times 10^{6} \mathrm{PFU} / \mathrm{ml}\right)$.

\section{Plaque assay}

The DENV-2 titer in the flies and mosquitoes was calculated using plaque assay method as described earlier ${ }^{29}$. The virus titer in the carcasses individual mosquito (virus blood fed or virus injected) or fly (virus injected) was reported as plaque forming units (PFU) (values are expressed as the mean $\pm \mathrm{SD}$ ).

\section{DENV-2 infection}

Infections were carried out by injecting $\sim 1 \mu$ of a viral suspension $\left(8.23 \times 10^{6} \mathrm{PFU} / \mathrm{ml}\right)$ into the thoraces of D. melanogaster adult flies $(\mathrm{n}=246)$ and Ae. aegypti $(\mathrm{n}=168)$. Actual injected titer of DENV-2 was determined using plaque assay at $2 \mathrm{~h}$ p.i.. Infected flies were then maintained at $24^{\circ} \mathrm{C}$. Ae. aegypti mosquitoes were infected with DENV-2 via blood through membrane feeder $(\mathrm{n}=76)$ and then maintained at $24^{\circ} \mathrm{C}$ for 11 days. To check infectious nature of DENV-2 produced in $D$. melanogaster, carcasses of DENV-2 positive flies were crushed and centrifuged at $4^{\circ} \mathrm{C}, 10000$ rpm, for $30 \mathrm{~min}$ and supernatant filtered through $0.22 \mu \mathrm{m}$ syringe filter and inoculated in $D$. melanogaster $(\mathrm{n}=94)$. The homogenates were mixed with blood and oral fed to Ae. aegypti $(\mathrm{n}=82)$. Antibodies

DENV-2 was inoculated in three-four weeks old Swiss albino mice intra-peritoneally and booster doses of DENV-2 were given (one dose/week) along with Freund's incomplete adjuvant (1:1) for two weeks. The mice were injected with $10 \%$ ascitic tumor cells intra-peritonealy. The intraperitoneal fluid collected and after removal of the debris by centrifugation, the supernatant was used to check for the presence of antibodies. Anti DENV-2 antibodies were incubated with the mosquito midgut extract to remove the non-specific antibodies. The protein-A column was used to purify the antibodies. The pre-immune serum was collected and checked for presence of non specific antibodies against the DENV-2. 
116 loading control [ab8224] (Abcam, USA), anti- tubulin antibody [T0950] (Sigma, USA),

117 Monoclonal Anti-Hsc70 (Hsp73) antibody [SAB3701436] (Sigma, USA), anti-HSP70 antibody

118 ([5A5] (ab2787), Abcam, USA) and anti-prohibitin antibody [EP2803Y] (ab75766) (Abcam, USA)

119 were used to confirm the results obtained in the various assays.

120 Detection of dengue viral antigen in the head squashes

121 The presence of viral antigen was determined by indirect immunofluroscence assay (IFA) in the

122 head squashes of mosquitoes/flies as described by Apte-Deshpande et $a .^{29}$. Along with each

123 experiment, positive and negative controls were processed using the same protocol. Presence of

124 DENV-2 antigen detected in head squash preparation of D. melanogaster flies on everyday till 10

125 days post infection (p.i.).

126 Membrane Fraction isolation

127 Brush-border membrane fractions (BBMF) from guts of Drosophila were isolated according to the 128 procedure described earlier ${ }^{2}$.

129 Virus Overlay Protein Binding Assay (VOPBA)

130 VOPBA was performed to identify cell polypeptides involved in virus binding following the

131 procedure described earlier ${ }^{2}$. Experiments were performed independently four times and negative

132 controls (without virus incubation, without antibody incubation) were kept. In VOPBA, interacting

133 proteins were identified, therefore no positive control is available for this assay.

\section{Protein identification using MALDI-TOF/TOF MS}

135 Bands corresponding to DENV-2-binding activity were excised from gels and were subjected to 136 reduction, alkylation, followed by in-gel digestion with trypsin. Extracted peptides were desalted 137 using the column and were separated on a Biobasic C18 capillary column. The chromatographically 138 seperated peptide masses were analyzed by matrix-assisted laser desorption/ionization time of flight 139 (MALDI-TOF/TOF) on Ultraflex TOF/TOF (Bruker Daltonics, Germany). The proteins were 140 identified using the mass spectrum produced from each sample by searching the $\mathrm{m} / \mathrm{z}$ values against 
141 the protein databases (NCBInr, MSDB, and Swissprot) using the MASCOT, MSfit and Profound

142 search engine. Parameters used for identification of proteins were fragment ion mass tolerance of

$1430.40 \mathrm{Da}$, parent ion tolerance of $0.4 \mathrm{Da}$ and iodoacetamide derivative of cysteine as a fixed

144 modification. The monoclonal anti-prohibitin antibody ([EP2803Y, ab75766, Abcam, USA), anti-

145 tubulin antibody (T0950 Sigma, Aldrich, Germany), anti-HSP70 antibody ([5A5] (ab2787), Abcam,

146 USA) were used to validate the results obtained in the MALDI TOF/TOF MS analysis.

\section{RNA isolation}

148 The QIAamp viral RNA mini kit (Qiagen USA) was used to isolate the viral RNA from carcasses of

149 flies. Total RNA was isolated from DENV-2 positive carcasses of Ae. Aegypti and D. melanogaster

150 flies using the RNA purification kit (Ambion-Thermo Fisher USA).

151 Detection of DENV by RT-PCR

152 Detection of DENV-2 in D. melanogaster and Ae. aegypti was performed using a RT-PCR

153 procedures described earlier ${ }^{30}$. The viral RNA was converted into cDNA using Goscript cDNA

154 synthesis system and the PCR amplification was performed in a Veriti thermocycler (Life

155 technology, USA). Negative controls consisted of RNA from uninfected Ae. aegypti and D.

156 melanogaster flies and water instead RNA. The second round of PCR was performed with $2 \mu 1$ of

157 sample from first round of amplification reaction.

158 Real-time qPCR assays

159 RNA samples $(2 \mu \mathrm{g})$ were incubated with Turbo DNase (Ambion, USA) and reverse-transcribed

160 using High capacity cDNA synthesis system (Life technologies, USA). Real-time relative

161 quantification of $50 \mathrm{ng}$ of cDNA was carried out using the Power SYBR Green PCR Kit (Life

162 technologies, USA) and ABI Detection System ABI 7300 (Applied Biosystems, USA). Four

163 independent biological replicates were conducted for each sample which were loaded in duplicates.

164 Primer sequences for Drosophila genes were retrieved from Flyprimerbank ${ }^{31}$ and are listed in Table

165 1. Fluorescence detection was performed at the end of each extension step and amplicon specificity

166 was checked by dissociation curve analysis at a rate of $1{ }^{\circ} \mathrm{C}$ every $30 \mathrm{~s}$ from 60 to $95^{\circ} \mathrm{C}$. All samples 
167

168

169

170

171

were amplified in duplicate from the same RNA sample and the mean value was calculated and was used for relative fold change analysis. The quantitative expression of the target gene was normalized to $18 \mathrm{~s}$ mRNA in the same samples.

\subsection{Statistical analysis}

The groups (control vs infected, Aedes aegypti DENV-2 injected vs Drosophila DENV-2 injected, 2 hrs p.i. vs 7 days p.i., fold change in control vs infected flies) were compared by nonparametric Mann-Whitney U test. The viral loads were log-transformed and were compared by non-parametric Mann-Whitney U test.

\section{RESULTS}

Does DENV-2 exploit similar molecules in different insects?

The studies on DENV interacting proteins in Aedes cells suggest that housekeeping molecules are exploited by DENV to establish the infection. Therefore, we identified the D. melanogaster gut BBMF proteins which are interacting with DENV-2 using one dimensional and two dimensional VOPBA and MALDI TOF/TOF analysis.

When immobilized brush border membrane fraction polypeptides were incubated with DENV-2, seven polypeptides, Belle, gamma-tubulin ring complex subunit, HSP 70Ba, ATP synthase subunit beta, probable tubulin beta chain, prohibitin and RNA recognition protein were detected as DENV-2 binding proteins in brush border membrane fraction of D. melanogaster (Fig. 1; Table 2, Supplementary Information Table 2). The identification of protein bands were further confirmed using monoclonal antibodies. DENV-2 interacting proteins documented earlier and the observations of current study suggest that Drosophila possesses the necessary molecules which could help DENV-2 in establishing the infection (Supplementary Information Table S1 for DENV binding proteins in insect cells). Therefore, it would be useful to test the susceptibility of $D$. melanogaster to DENV-2 by injecting non lethal dose of DENV-2 in thoracic region of adult flies [Exposure of D. melanogaster flies to a low dose of DENV-2 $\left(\sim 1 \mu \mathrm{l}\right.$ of $\left.8.23 \times 10^{6} \mathrm{PFU} / \mathrm{ml}\right)$ did not 
193 have an effect on life span nor increased mortality compared to controls Logrank test $P=0.73$ data

194 not shown].

195 Susceptibility of $\underline{D}$. melanogaster to DENV-2

196 To determine the DENV-2 susceptibility, a non lethal dose of DENV-2 virus $\left(\sim 1 \mu 1\right.$ of $8.23 \times 10^{6}$

$197 \mathrm{PFU} / \mathrm{ml}$ ) was injected in thoracic region of adult Drosophila flies. DENV-2 was detected in $D$.

198 melanogaster midgut, brain and carcasses every day till 10 days p.i.. Immuno-fluorescence

199 microscopy showed the presence of DENV-2 in D. melanogaster brain after 7 days of post infection

200 (Fig. 2A). Detection of virus in gut tissue in addition to its time dependent appearance in brain

201 indicated propagation of virus in the body of D. melanogaster flies (Fig. 2A). RT-PCR results

202 showed the presence of DENV-2 in carcasses of D. melanogaster (Fig. 2B). Sequencing of RT-PCR

203 product confirmed the specificity of RT-PCR. Susceptibility of D. melanogaster (61\% \pm 6.4$)$ to

204 DENV-2 at 7 days p.i. was found to be comparable to Ae. aegypti (72\% \pm 13$)$ (Mann-Whitney U test

$205 P>0.05)($ Fig. 2C).

206 Determination of infectivity of $\underline{D}$. melanogaster generated DENV-2

207 In order to determine infectious nature of DENV-2 produced in D. melanogaster, both $D$. 208 melanogaster and Ae. aegypti were infected with homogenates of DENV-2 virus positive flies. In 209 orally fed Ae. aegypti, the DENV-2 antigen was detected in head squash preparations after 11 days 210 (24\%), while $56 \%$ of inoculated D. melanogaster showed presence of DENV-2 in brain after 7 211 days (Fig. 2C). These data not only confirmed the D. melanogaster susceptibility to DENV-2, but 212 also demonstrated that the infectious nature of D. melanogaster generated DENV-2. These 213 experiments were repeated several times with reproducible results.

214 DENV-2 quantitation using plaque assay

215 Plaque assays were used to determine the multiplication of DENV-2 in Ae. Aegypti and D. 216 melanogaster. Ae. aegypti mosquitoes were infected with DENV-2 by oral feeding and intra217 thoracic inoculation and D. melanogaster flies were infected intra thoracically with DENV-2 and 218 were maintained at $28^{\circ} \mathrm{C}$ for 7 days. The DENV-2 titers in the carcasses of DENV-2 positive insects 
were determined by plaque assay (Fig. 2D). No significant difference was observed in DENV-2

220 viral load at 2 hrs p.i. in DENV-2 inoculated Ae. aegypti (5408 $\pm 862 \mathrm{PFU} / \mathrm{mosquito})$ and $D$.

221 melanogaster (4511 \pm 968 PFU/fly) (Mann-Whitney U test $P>0.05$ ). These observations suggest that

222 similar dose of DENV-2 was given to Ae. aegypti and D. melanogaster. At 7 days p.i., as compared

223 to 2 h p.i., DENV-2 viral load was significantly increased in Ae. aegypti $(85000 \pm 2684$

224 PFU/mosquito) (Mann-Whitney U test $P<0.05$ ) and D. melanogaster (56983 \pm 8962 PFU/fly)

225 (Mann-Whitney U test $P<0.05$ ). The homogenates of DENV-2 positive $D$. melanogaster was used

226 to infect D. melanogaster and Ae. aegypti. At 7 days p.i., as compared to $2 \mathrm{~h}$ p.i., the DENV-2 viral

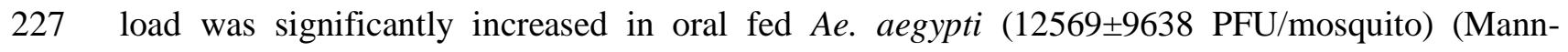

228 Whitney U test $P<0.05)$ and intra thoracic injected D. melanogaster $(26982 \pm 12692$ PFU/fly)

229 (Mann-Whitney $U$ test $P<0.05$ ). At 7 days p.i., the virus titer in intra thoracic injected $D$.

230 melanogaster and Ae. Aegypti flies was almost similar (Mann-Whitney U test $P>0.05$ ).

231 Anti-DENV-2 response in $\underline{\text { D. }}$ melanogaster

232 The antiviral response of $D$. melanogaster against the DENV-2 virus infection was checked in 233 selected antiviral pathway genes using the qPCR assays. The expression levels of key components 234 of JAK-STAT, RNA interference (RNAi) and Toll pathway were assessed in DENV-2 infected $D$. 235 melanogaster using $\mathrm{qPCR}$ at $24 \mathrm{~h}, 48 \mathrm{~h}$ and 7 days p.i.. DENV-2 stimulates the transcriptional 236 activation of RNAi, JAK-STAT and Toll pathway (Table 3). Transcript levels of key mediators of 237 RNAi pathway, Argonaute 1, Argonaute 2, Argonaute 3, Dicer 1, Dicer 2, Drosha and Pasha were 238 up-regulated during course of DENV-2 infection (Mann-Whitney $\mathrm{U}$ test $P<0.05$ ). ADAR, FMR, 239 Loqs, RNBP and VIG showed slight variation in transcript levels in response to DENV-2 infection 240 (Table 3). Transcript levels of JAK, STAT, Prohitibin, Rel1 and Toll were up-regduring DENV-2 241 infection (Table 3) (Mann-Whitney U test $P<0.05$ ).

\section{DISCUSSION}



melanogaster as well as its potential to use as a test system to analyze human diseases ${ }^{12-18}$, makes this non vector dipteran as an attractive model to study the pathogens and propagation in these 247 pathogens in the insect model systems ${ }^{19-24,27}$. Using genome-wide RNA interference screen in $D$. 248 melanogaster the insect host factors required for DENV propagation were identified ${ }^{25}$. The results 249 of Sessons et al. $^{25}$ study indicate that remarkable conservation in required factors between the 250 dipteran and human hosts. Recently it has been demonstrated that RNA interference modulates the 251 replication of dengue virus in D. melanogaster cells ${ }^{25,32}$. Results obtained in these studies clearly 252 suggest that DENV could propagate in Drosophila cells. Recently Rences et al. ${ }^{33,34}$ utilized the $D$. 253 melanogaster and Ae. aegypti to understand the molecular mechanisms involved in Wolbachia254 mediated pathogen protection. The infection with Wolbachia efficiently reduced the DENV 255 replication in D. melanogaster as well as in Ae. aegypti ${ }^{33,34}$. The results obtained in these studies 256 demonstrated that the mechanism of DENV blocking by Wolbachia is more complicated than a 257 simple priming of the insect innate immune system. It will be interesting to investigate the complex 258 mechanism involved in DENV-vector interactions. Availability of Drosophila mutants will help in 259 deciphering the complex interactions involved in DENV infection. The binding proteins, 260 susceptibility and virus titer of DENV-2 in D. melanogaster was not investigated in earlier studies. 261 We, therefore, infected D. melanogaster with DENV-2 and found that D. melanogaster was not 262 only susceptible to DENV-2 but also produced infectious DENV-2 particles. The DENV-2 infection 263 in D. melanogaster seems to be a specific pathogenic process rather than nonspecific viremia. First, 264 DENV-2 was detected in midgut, carcasses and brain after 7 days of post infection period in $D$. 265 melanogaster similar to in Ae. aegypti. Second, DENV-2 virus produced in fly is equally infectious 266 to both $D$. melanogaster and Ae. aegypti. Third, after 7 days post infection DENV-2 titers were 267 comparable in Ae. aegypti and D. melanogaster. These qualities make D. melanogaster a potential 268 model system for examining DENV-vector interactions. The Drosophila model system is useful in 269 rapid and unbiased identification of host factors involved in pathogenesis ${ }^{12,14,19,26,27}$. Considering the 
270 biosafety issues, the fruit fly system has certain added advantages in comparison with the

271 anthropogenic vectors such as mosquito, sand fly and tick.

272 DENV-2 binding proteins in mosquito cells have been identified in several studies ${ }^{2,3,5,6}$ [see

273 supplementary Information Table 1]. However, the identity of most of the proteins is not

274 conclusively determined. Proteins such as HSP 70Ba, ATP synthase sub unit beta, probable tubulin

275 beta chain and prohibitin were found interacting with Ae. aegypti and D. melanogaster. Based on

276 results obtained in the previous studies and current study, it is reasonable to infer that DENV-2 may

277 share the similar receptor molecule(s) on dipteran cells ${ }^{2,3,5}$. HSP 70 proteins might be binding

278 receptor and other proteins such as actin, tubulin, ATP synthase etc. might be secondary receptors.

279 Although in most cases, individual viruses have their own distinct and specific receptors, in some

280 cases the same set of receptors can be used by many different viruses. Japanese Encephalitis Virus

281 (JEV), DENV and West Nile Virus (WNV) share the similar molecules for their entry into the

282 mosquito cells ${ }^{2,3,5,6,35,36}$. Perhaps the best studied example of this is the HSP 70 of Ae. aegypti,

283 which is used by DENV, JEV and WNV as a receptor ${ }^{2,5,6,7}$. As these molecules are house keeping in

284 nature, they are present in other dipteran insects also. We hypothesize that DENV-2 might share

285 similar receptors in dipteran insects such as Ae. aegypti, Ae. albopictus and D. melanogaster.

Interestingly in VOPBA experiments, Belle and RNA recognition protein were recognized

287 as DENV-2 binding proteins. In Drosophila, Belle, a DEAD-box RNA helicase, has been 288 documented to regulate RNA interference (RNAi) ${ }^{37}$. During the infection, interaction and co289 localization of DDX3 (a human homologue of Belle) with arboviral proteins and viral RNA have 290 been demonstrated ${ }^{38,39}$. Similarly, the role of RRM proteins in the post-transcriptional gene 291 expression modulation of the Drosophila RNAi pathway is well documented ${ }^{37}$. These observations 292 hint the possible involvement of Drosophila RNAi pathway in controlling the DENV-2 infection. 293 Prohibitins (PHBs) are highly conserved proteins in eukaryotes and are associated with various 294 cellular functions including the immune regulation. Role of prohibitin as a non-receptor interacting 295 polypeptide in DENV-2 infection has been reported previously ${ }^{2,29}$. These observations suggest that 
similar immune response might be triggered in Ae. aegypti and D. melanogaster to counteract the

297 DENV-2 infection.

In the mosquito, arboviruses are confronted with RNAi, JAK-STAT and Toll pathway ${ }^{10-}$

12,40-49. RNAi is one of the important innate antiviral pathways in Ae. aegypti that controls the

300 arbovirus replication ${ }^{41,42,44-47,49-58}$. The recent reports demonstrated that RNAi pathway can

301 eliminate the DENV from Ae. aegypti and Dcr-2 and Ago-2 knockdowns enhances the DENV

302 replication $^{42,45,47,49}$. Similarly it has been also demonstrated that DENV can grow in Drosophila S2

303 cells and the RNAi regulates the DENV replication in these cells ${ }^{32}$. Therefore, we employed the

304 Drosophila RNAi, JAK-STAT and Toll gene expression to address how a model innate system

305 responds to DENV-2 infection. Transcript levels of most of the key mediator of RNAi response

306 were up-regulated in response to DENV-2 infection at 7 days p.i.. Moderate modulation in RNAi

307 pathway genes was observed at early time points $24 \mathrm{hrs}$ and $48 \mathrm{hrs}$ p.i.. Loqs expression was

308 slightly down-regulated in response to DENV-2 infection. It has been demonstrated that dsRNA of

309 viruses can be cleaved by Dcr-2 without Loqs-PD and complete knock down of Loqs-PD has no

310 effect on antiviral silencing ${ }^{37,59}$. These observations suggest that Loqs have limited role in antiviral

311 response in Drosophila.

312 Recent transcriptome analysis of DENV2-infected Ae. aegypti reveled the involvement

313 miRNA pathway in virus infection ${ }^{51-53}$. The significant increase in unique miRNAs was observed

314 during DENV infection in Ae. aegypti ${ }^{58}$. Over the course of infection, 9 days p.i. time point showed

315 maximum number of unique modulated miRNAs ${ }^{58}$. It has been suggested at 9 days p.i. the repair

316 mechanisms in uninfected mosquitoes is activated and results in to significant increase in the

317 miRNA levels at time point ${ }^{58}$. The expression of Argonate-1, Dicer-1, Drosha and Pasha increased

318 during the time course of DENV infection in D. melanogaster. These observations suggest that

319 miRNA pathway activity is altered during DENV-2 infection in D. melanogaster. The results

320 obtained in current study corroborate with earlier studies that showed the presence of modulated

321 miRNAs in DENV2 infection in mosquitoes. 
A significant role of piRNA pathway has been also reported in various arbovirus infections $^{54,55,57}$. It has been envisaged that a non-canonical piRNA pathway play important role in 324 vector mosquitoes and target alphavirus replication ${ }^{54}$. The activity PIWI proteins and virus-specific 325 piRNA molecules is also detected in somatic cells of D. melanogaster suggesting that the piRNA 326 pathway plays important role in antiviral functions in insects ${ }^{50}$. Induction in transcript levels of 327 Argonaute 3 AUB and PIWI suggest that DENV-2 replication is able to trigger the piRNA pathway. 328 The miRNA, piRNA and siRNA pathways may act in amalgamation to control DENV-2 infection in D. melanogaster. Toll pathway and JAK-STAT pathway (Table 3). In Ae. aegypti, the JAK-STAT and Toll pathway 332 are involved in the anti-dengue defense ${ }^{10}$. Up-regulation of the Toll pathway has also been reported 333 in Ae. aegypti in response to Sindbis virus infection ${ }^{40}$. The fruit fly seems to rely on RNAi, JAK334 STAT and Toll to counteract DENV-2 infections. Though the JAK-STAT, RNAi and Toll pathways 335 were seen to be induced in response to DENV infection, $60 \%$ of flies were still infected by DENV336 2. These observations suggest that JAK-STAT, RNAi and Toll pathway are activated in response to 337 DENV-2 infection but are not sufficient for complete elimination of DENV-2 in Drosophila. 338 DENV-2 must have evolved strategies to counteract the effects of the JAK-STAT, RNAi and Toll 339 pathway. In depth studies on host factors involved in virus infection is necessary to design and 341 develop effective intervention strategies. In many other insect vectors such as ticks, sand flies, fleas 342 and other members of Culicidae family, amenable genetic systems limit in depth understanding of 343 vector parasite interaction in these species. In this context, Drosophila becomes an attractive model 344 system to elucidate the complex host-parasite interactions. Drosophila possesses the necessary 345 repertoire of proteins that might require for virus entry. D. melanogaster supports the growth of 346 WNV, SINV and DENV-2. Further the immune response mounted against arboviruses is similar in 347 D. melanogaster and Ae. aegypti. Due to whole genome sequence and established techniques for 
genetic and developmental manipulations, D. melanogaster turn out to be an attractive model

organism to understand molecular and cellular mechanisms in host-arbovirus interactions. D.

350 melanogaster could be used as surrogate invertebrate host model system and can be used to study

351 parasite-vector interactions in less characterized vectors such as ticks, sand flies, fleas and other

352 Culicine mosquitoes.

\section{CONCLUSION}

In conclusion, VOPBA revealed that DENV-2 exploit similar molecules in D. melanogaster and Ae. aegypti for its entry. D. melanogaster supports the growth of DENV-2 virus and $D$.

356 melanogaster generated DENV-2 was able to infect the Ae. aegypti with similar kinetics. Results 357 obtained in this study and earlier reports suggest that D. melanogaster and Ae. aegypti mount 358 similar immune response against the invading arboviruses. These qualities make D. melanogaster, a 359 potential model system for the study of arbovirus-vector interactions.

ACKNOWLEDGEMENTS

362 We thank the Director, National Institute of Virology, Pune for the providing the facilities. We 363 thank Dr. Dipankar Chaterjee, Indian Institute of Science, Bangalore for MALDI TOF/TOF 364 analysis. This research was supported by UGC-CAS to Department of Zoology, University of Pune 365 and ICMR grant to Prof. Dileep Deobagkar.

\section{CONFLICT OF INTEREST}

368 The authors have declared that no competing interests exist.

FINANCIAL DISCLOSURE

371 The authors would like to acknowledge financial support provided by the UGC-CAS and ICMR.

\section{REFERENCES}


1. LaCount DJ, Vignali M, Chettier R, Phansalkar A, Bell R, Hesselberth JR, et al. A protein interaction network of the malaria parasite Plasmodium falciparum. Nature 2005; 438: 103-7.

2. Paingankar MS, Gokhale MD, Deobagkar DN. Dengue-2-virus-interacting polypeptides involved in mosquito cell infection. Arch Virol 2010; 155: 1453-61.

3. Kuadkitkan A, Wikan N, Fongsaran C, Smith DR. Identification and characterization of prohibitin as a receptor protein mediating DENV-2 entry into insect cells. Virology 2010;406: 149-61.

4. Demogines A, Abraham J, Choe H, Farzan M, Sawyer SL. Dual Host-Virus Arms Races Shape an Essential Housekeeping Protein. PLoS Biol 2013; 11(5): e1001571.

5. Paingankar MS and Arankalle VA. Identification of chikungunya virus interacting proteins in mammalian cells. J Biosci 2014; 39: 389-99.

6. Chu JJ, Leong PW, Ng ML. Characterization of plasma membrane-associated proteins from Aedes albopictus mosquito (C6/36) cells that mediate West Nile virus binding and infection. Virology 2005; 339(2): 249-60.

7. Ren J, Ding T, Zhang W, Song J, Ma W. Does Japanese encephalitis virus share the same cellular receptor with other mosquito-borne flaviviruses on the C6/36 mosquito cells? Virol J

8. Schneider D and Shahabuddin M. Malaria parasite development in a Drosophila model. Science 2000; 288: 2376-79.

9. Matthews BJ, Dudchenko O, Kingan SB, Koren S, Antoshechkin I, Crawford JE. Improved reference genome of Aedes aegypti informs arbovirus vector control. Nature 2018; 563: 501507.

10. Xi Z, Ramirez JL, Dimopoulos G. The Aedes aegypti Toll Pathway Controls Dengue Virus Infection. PLoS Pathog 2008; 4(7): e1000098. pathway in anti-dengue defense. Proc Natl Acad Sci USA 2009;106: 17841-6. 
400

401

402

403

404

405

406

407

408

409

410

411

412

413

414

415

416

417

418

419

420

422

424

425

12. Waldock J, Olson KE, Christophides GK. Anopheles gambiae Antiviral Immune Response to Systemic O'nyong-nyong Infection. PLoS Negl Trop Dis 2012; 6(3): e1565.

13. Bonini NM and Fortini ME. Human neurodegenerative disease modeling using Drosophila. Ann Rev Neurosci 2003; 26: 627-656.

14. Dionne MS, Ghori N, Schneider DS. Drosophila melanogaster is a genetically tractable model host for Mycobacterium marinum. Infect Immun 2003;71: 3540-50.

15. Bier E. Drosophila, the golden bug, emerges as a tool for human genetics. Nat Rev Genet 2005; 6: 9-23.

16. Pandey UB and Nichols CD. Human disease models in Drosophila melanogaster and the role of the fly in therapeutic drug discovery. Pharmacol Rev 2011;63(2): 411-36.

17. Park SY, Ludwig MZ, Tamarina NA, He BZ, Carl SH, Dickerson DA, et al. Genetic Complexity in a Drosophila Model of Diabetes-Associated Misfolded Human Proinsulin. Genetics, 2013;196(2): 539-55.

18. Tutor AS, Prieto-Sánchez S, Ruiz-Gómez M. Src64B phosphorylates Dumbfounded and regulates slit diaphragm dynamics: Drosophila as a model to study nephropathies. Development 2014;141(2): 367-76.

19. Mansfield BE, Dionne MS, Schneider DS, Freitag NE. Exploration of host-pathogen interactions using Listeria monocytogenes and Drosophila melanogaster. Cell Microbiol 2003; 5: $901-11$.

20. Needham AJ, Kibart M, Crossley H, Ingham PW, Foster SJ. Drosophila melanogaster as a model host for Staphylococcus aureus infection. Microbiology 2004; 150: 2347-55.

21. Blow NS, Salomon RN, Garrity K, Reveillaud I, Kopin A, et al. Vibrio cholerae infection of Drosophila melanogaster mimics the human disease cholera. PLoS Pathog 2005; 1(1): e8.

22. Garcia-Lara J, Needham AJ, Foster SJ. Invertebrates as animal models for Staphylococcus aureus pathogenesis: A window into host-pathogen interaction. FEMS Immunol Med Microbiol 2005; 43: 311-23. 
23. Chotkowski HL, Ciot AT, Jia Y, Puig-Basagoitic F, Kramer LD, Shi PY and Glaser RL. West Nile Virus Infection of Drosophila melanogaster induces a Protective RNAi Response. Virology 2008; 377: 197-206.

24. Carpenter J, Hutter S, Baines JF, Roller J, Saminadin-Peter SS, et al. The Transcriptional Response of Drosophila melanogaster to Infection with the Sigma Virus (Rhabdoviridae). PLoS ONE 2009; 4(8): e6838.

25. Sessions OM, Barrows NJ, Souza-Neto JA, Robinson TJ, Hershey CL, Rodgers MA, et al. Discovery of insect and human dengue virus host factors. Nature 2009; 458: 1047-50.

26. Hughes TT, Allen AL, Bardin JE, Christian MN, Daimon K, Dozier KD, et al. Drosophila as a genetic model for studying pathogenic human viruses. Virology 2012; 423(1): 1-5.

27. Shaw AE, Veronesi E, Maurin G, Ftaich N, Guiguen F, Rixon F, et al. Drosophila melanogaster as a Model Organism for Bluetongue Virus Replication and Tropism. J Virol 2012; 86(17):9015-24. doi: 10.1128/JVI.00131-12.

28. Querenet M, Danjoy ML, Mollereau B, Davoust N. Expression of dengue virus NS3 protein in Drosophila alters its susceptibility to infection. Fly (Austin) 2015;9(1):1-6. doi: 10.1080/19336934.2015.1072662.

29. Apte-Deshpande A, Paingankar MS, Gokhale MD and Deobagkar DN. Serratia odorifera a midgut inhabitant of Aedes aegypti mosquito enhances its susceptibility to dengue-2 virus. PLoS ONE 2012; 7(7): e40401.

30. Lanciotti RS, Calisher CH, Gubler DJ, Chang GJ, Vance vorndam A. Rapid detection and typing of dengue viruses from clinical samples by using reverse transcriptase-polymerase chain reaction. J Clin Microbiol 1992; 30(3): 545-551.

31. Hu Y, Sopko R, Foos M, Kelley C, Flockhart I, Ammeux N, et al. FlyPrimerBank: an online database for Drosophila melanogaster gene expression analysis and knockdown evaluation of RNAi reagents. G3: Genes Genomes Genetics 2013; 3(9): 1607-16. 
32. Mukherjee S and Hanley KA. RNA interference modulates replication of dengue virus in Drosophila melanogaster cells. BMC Microbiol 2010; 10: 127.

33. Rancès E, Ye YH, Woolfit M, McGraw EA, O'Neill SL. The relative importance of innate immune priming in Wolbachia-mediated dengue interference. PLoS Pathog 2012; 8(2): e1002548.

34. Rancès E, Johnson TK, Popovici J, Iturbe-Ormaetxe I, Zakir T, Warr CG, et al. The toll and Imd pathways are not required for wolbachia-mediated dengue virus interference. J Virol 2013; 87(21): 11945-9.

35. Boonsanay V and Smith DR. Entry into and production of the Japanese encephalitis virus from C6/36 cells. Intervirol 2007; 50(2): 85-92.

36. Vega-Almeida TO, Salas-Benito M, De Nova-Ocampo MA, del Angel RM, Salas-Benito JS. Surface proteins of C6/36 cells involved in dengue virus 4 binding and entry. Arch Virol 2013; 158 (6): 1189-1207.

37. Meister G. Argonaute proteins: functional insights and emerging roles. Nature Rev Genet 2013; 14: 447-59.

38. Khadka S, Vangeloff AD, Zhang C, Siddavatam P, Heaton NS, Wang L, et al. A physical interaction network of dengue virus and human proteins. Mol Cell Proteom 2011; 10(12): M111-012187.

39. Li C, Ge LL, Li PP, Wang Y, Dai JJ, Sun MX et al. Cellular DDX3 regulates Japanese encephalitis virus replication by interacting with viral un-translated regions. Virology 2014; 449: $70-81$.

40. Sanders HR, Foy BD, Evans AM, Ross LS, Beaty BJ, et al. Sindbis virus induces transport processes and alters expression of innate immunity pathway genes in the midgut of the disease vector, Aedes aegypti. Insect Biochem Mol Biol 2005; 35: 1293-1307.

41. Campbell CL, Keene KM, Brackney DE, Olson KE, Blair CD, Wilusz J, et $\square$ al. Aedes aegypti uses RNA interference in defense against Sindbis virus infection. BMC Microbiol 2008; 8: 47. 
42. Sanchez-Vargas I, Scott JC, Poole-Smith BK, Franz AWE, Barbosa-Solomieu V, Wilusz J, et $\square$ al. Dengue virus type 2 infections of Aedes aegypti are modulated by the mosquito's RNA interference pathway. PLOS Pathog 2009; 5: e1000299.

43. Ramirez JL and Dimopoulos G. The Toll immune signaling pathway control conserved antidengue defenses across diverse Ae. aegypti strains and against multiple dengue virus serotypes. Dev Comp Immunol 2010; 34: 625-29.

44. Khoo CCH, Piper J, Sanchez-Vargas I, Olson KE, Franz AWE. The RNA interference pathway affects midgut infection- and escape barriers for Sindbis virus in Aedes aegypti. BMC Microbiol 2010;10: 130 .

45. Scott JC, Brackney DE, Campbell CL, Bondu-Hawkins V, Hjelle B, Ebel GD, et $\square$ al. Comparison of dengue virus type 2-specific small RNAs from RNA interference-competent and-incompetent mosquito cells. PLOS Negl Trop Dis 2010; 4: e848.

46. Siu R, Fragkoudis R, Simmonds P, Donald CL, Chase-Topping ME, Barry G, et $\square$ al. Antiviral RNA interference responses induced by Semliki Forest virus infection of mosquito cells: characterization, origin, and frequency-dependent functions of virus-derived small interfering RNAs. J Virol 2011; 85: 2907-17.

47. Hess AM, Abhishek NP, Ptitsyn A, Ebel GD, Olson KE, Barbacioru C, et $\square$ al. Small RNA profiling of dengue virus-mosquito interactions implicates the PIWI RNA pathway in anti-viral defense. BMC Microbiol 2011; 11: 45.

48. Blair CD. Mosquito RNAi is the major innate immune pathway controlling arbovirus infection and transmission. Future Microbiol 2011; 6: 265-77.

49. Khoo CC, Doty JB, Heersink MS, Olson KE, Franz AW. Transgene-mediated suppression of the RNA interference pathway in Aedes aegypti interferes with gene silencing and enhances Sindbis virus and dengue virus type 2 replication. Insect Mol Biol 2013; 22(1): 104-14. 
50. Wu Q, Luo Y, Lu R, Lau N, Lai EC, Li WX, et al. Virus discovery by deep sequencing and assembly of virus-derived small silencing RNAs. Proc Natl Acad Sci USA 2010; 107(4): 16061611.

51. Behura SK, Gomez-Machorro C, Harker BW, deBruyn B, Lovin DD, et al. Global Cross-Talk of Genes of the Mosquito Aedes aegypti in Response to Dengue Virus Infection. PLoS Negl Trop Dis 2011; 5(11): e1385.

52. Colpitts TM, Cox J, Vanlandingham DL, Feitosa FM, Cheng G, et al. Alterations in the Aedes aegypti Transcriptome during Infection with West Nile, Dengue and Yellow Fever Viruses. PLoS Pathog 2011; 7 (9): e1002189.

53. Bonizzoni M, Dunn WA, Campbell CL, Olson KE, Marinotti O, et al. Complex Modulation of the Aedes aegypti Transcriptome in Response to Dengue Virus Infection. PLoS ONE 2012; 7(11): e50512.

54. Morazzani EM, Wiley MR, Murreddu MG, Adelman ZN, Myles KM. Production of VirusDerived Ping-Pong-Dependent piRNA-like Small RNAs in the Mosquito Soma. PLoS Pathog 2012; 8(1): e1002470.

55. Vodovar N, Bronkhorst AW, van Cleef KWR, Miesen P, Blanc H, et al. Arbovirus-Derived piRNAs Exhibit a Ping-Pong Signature in Mosquito Cells. PLoS ONE 2012; 7(1): e30861.

56. Marques JT, Wang JP, Wang X, de Oliveira KPV, Gao C, et al. Functional Specialization of the Small Interfering RNA Pathway in Response to Virus Infection. PLoS Pathog 2013; 9(8): e1003579.

57. Schnettler E, Donald CL, Human S, Watson M, Siu RWC, McFarlane M, et al. Knockdown of piRNA pathway proteins results in enhanced Semliki Forest virus production in mosquito cells. J Gen Virol 2013; 94: 1680-89.

58. Campbell CL, Harrison T, Hess AM and Ebel GD. MicroRNA levels are modulated in Aedes aegypti after exposure to Dengue-2. Insect Mol Biol 2014; 23(1): 132-9. 
bioRxiv preprint doi: https://doi org/10.1101/2020.09.03.282350; this version posted September 4, 2020. The copyright holder for this preprint

(which was not certified by peer review) is the author/funder, who has granted bioRxiv a license to display the preprint in perpetuity. It is made available under aCC-BY-NC-ND 4.0 International license.

526 59. Marques JT, Kim K, Wu PH, Alleyne TM, Jafari N, et al. Loqs and R2D2 act sequentially in the

$527 \quad$ siRNA pathway in Drosophila. Nat Struct Mol Biol 2010; 17: 24-30.

528 
530 Fig. 1. DENV-2 binding proteins in D. melanogaster. A) Membrane proteins from D. 531 melanogaster midgut (lane L1, lane L2 and lane L3) were subjected to SDS-12.5\% PAGE, 532 transferred to nitrocellulose membrane, and incubated with DENV-2 (lanes L1, lane L2) and PBS 533 (lane L3) at $37^{\circ} \mathrm{C}$ for 1 hour. The putative Dengue virus interacting proteins revealed after 534 incubation with the anti-dengue-2 rabbit antibody (Lane L1 and Lane L3) and with a second 535 antibody, an antibody mouse anti rabbit IgG conjugated to peroxidase. Lane B was only incubated 536 with secondary antibody. Color was developed with $\mathrm{H}_{2} \mathrm{O}_{2}$ and DAB. B) Verification of MALDI 537 TOF/TOF results using monoclonal antibodies Membrane proteins were subjected to $12.5 \%$ SDS 538 PAGE and transferred to nylon membrane. Anti HSP70 antibody, Anti tubulin antibody and anti 539 prohibitin antibody detected the bands corresponding to $70 \mathrm{kDa}, 48 \mathrm{kDa}$ and $32 \mathrm{kDa}$ respectively. 540 Anti actin antibody detected band corresponding to $42 \mathrm{kDa}$. However, $42 \mathrm{kDa}$ band was not 541 detected in VOPBA.

543 Fig. 2. Susceptibility of $\boldsymbol{D}$. melanogaster to DENV-2. A) Detection of DENV-2 in head squash of 544 D. melanogater and Ae. aegypti: D. melanogaster and Ae. aegypti were intra thoracically injected 545 with DENV-2 (3-4 $\mu$ l of $\left.10^{6} \mathrm{PFU} / \mathrm{ml}\right)$ or PBS (pH 7.4). DENV-2 was detected in head squash at 7 546 days p.i. using immuno-fluorescence microscopy. DENV-2 stained with FITC conjugated antibody 547 (green color) and the neural tissue mass with Evan's Blue (red color). I) D. melanogaster head 548 squash, II) A. aegypti mosquitoes flies III) Midgut of D. melanogaster. B) RT-PCR detection of 549 DENV-2: DENV-2 detected in carcasses of D. melanogaster and Ae. aegypti using RT-PCR. 1) 550 Uninfected Ae. aegypti 2) Uninfected D. melanogaster 3) Oral infection DENV-2 in Ae. aegypti 4) 551 intra thoracic injection of DENV-2 in Ae. aegypti 5) intra thoracic injection of DENV-2 in $D$. 552 melanogaster 6) DNA ladder 100bp 7) intra thoracic injection of homogenate of DENV-2 positive 553 D. melanogaster in Ae. aegypti 8) oral feeding of homogenate of DENV-2 positive D. melanogaster 554 in Ae. aegypti 9) intra thoracic injection of homogenate of DENV-2 positive D. melanogaster in D. 
555 melanogaster 10) non template control (NTC). C) Ae. aegypti and D. melanogaster susceptibility to

556 DENV-2: Percent DENV-2 positive head squash preparations of mosquitoes and flies were detected

557 by immunofluorescence assay. DENV-2 inoculated Ae. Aegypti and D. melanogaster flies showed 558 similar pattern of DENV-2 susceptibility (Mann-Whitney U test $P>0.05$ ) D) DENV-2 quantitation 559 using plaque assay: DENV-2 titers in carcasses of individual D. melanogaster and Ae. aegypti at 2

560 hrs p.i. and 7 days p.i were quantitated using plaque assay. * significant difference in plaque forming 561 units as compared to $2 \mathrm{hrs}$ p.i..

562

563 Supplementary Information Table S1: DENV-2 interacting proteins in insect cells

564

565 Supplementary Information Table S2: Molecular identification of DENV-2 binding proteins

566 from $D$. melanogaster midgut using 1D and 2D VOPBA 
Table 1: Primers used for real time PCR

\begin{tabular}{|c|c|c|c|c|c|c|c|}
\hline & $\begin{array}{l}\text { Gene } \\
\text { Name }\end{array}$ & Gene ID & Forward Primer & $\mathrm{Tm}$ & Reverse Primer & $\mathrm{Tm}$ & $\begin{array}{l}\text { Product } \\
\text { size }\end{array}$ \\
\hline 1 & $18 \mathrm{~s}$ & KC177303 & ATAAGACCTCTGTTCTGCTTTC & $60^{\circ} \mathrm{C}$ & CTCTCGCGTCGTAATACTAATG & $60^{\circ} \mathrm{C}$ & 92 \\
\hline 2 & ADAR & CG12598 & TGCTGAATGAGCTAAGACATGG & $60.1^{\circ} \mathrm{C}$ & TGAATAGAGGTGCGTGTACCG & $61.6^{\circ} \mathrm{C}$ & 75 \\
\hline 3 & Ago1 & CG6671 & GTGTCCGCGAAAGGTGAAC & $61.3^{\circ} \mathrm{C}$ & AGATTGTTGCGACCATCGAAC & $61^{\circ} \mathrm{C}$ & 102 \\
\hline 4 & Ago2 & $\begin{array}{l}\text { CG13452, } \\
\text { CG7439 }\end{array}$ & TTCGACAAGCCCATGCGAG & $62.7^{\circ} \mathrm{C}$ & AAAGAAGGAACGACCGACACG & $62.6^{\circ} \mathrm{C}$ & 93 \\
\hline 5 & Ago3 & CG40300 & AGCTAACGACGGATGAATCCA & $61^{\circ} \mathrm{C}$ & TGGTTCCACCGAGTTTATCCT & $60.5^{\circ} \mathrm{C}$ & 123 \\
\hline 6 & Aub2 & CG6137 & TTACGCCTGATGTGGAGGCTA & $62.7^{\circ} \mathrm{C}$ & GGGGCTATCTTGAACAGCTTTG & $61.2^{\circ} \mathrm{C}$ & 134 \\
\hline 7 & Dicer1 & CG4792 & TTCCACTGGTGCGACAACAAT & $62.5^{\circ} \mathrm{C}$ & CCCAGGCAAATAATCGTGTTCC & $61.5^{\circ} \mathrm{C}$ & 107 \\
\hline 8 & Dicer2 & CG6493 & GCTTTTATGTGGGTGAACAGGG & $61.4^{\circ} \mathrm{C}$ & GGCTGTGCCAACAAGAACTT & $61.1^{\circ} \mathrm{C}$ & 92 \\
\hline 9 & Drosha & CG8730 & GGAGACACCGGCTCCTTATG & $61.7^{\circ} \mathrm{C}$ & ACTTCTGTGCTTGTTCGTTGTAA & $60.6^{\circ} \mathrm{C}$ & 107 \\
\hline 10 & FMR1 & CG6203 & GCCAATTACAGCCAAGACCTT & $60.6^{\circ} \mathrm{C}$ & CGCTTCTGAGTGTGCTCAAAC & $61.8^{\circ} \mathrm{C}$ & 183 \\
\hline 11 & JAK & CG1594 & AGGATTTCCTCAATCGCCTT & $58^{\circ} \mathrm{C}$ & CAGCTGCATCAGGTCGTAAA & $60^{\circ} \mathrm{C}$ & 86 \\
\hline 12 & Loqs & CG6866 & AGCGCCATGTGAAGCTCAA & $62.3^{\circ} \mathrm{C}$ & CAGGATCACCAACATCTGACAG & $60.4^{\circ} \mathrm{C}$ & 96 \\
\hline 13 & Pasha & CG1800 & AAGTCCTACCCGAGGGATGG & $62.3^{\circ} \mathrm{C}$ & TCCAGTGCCGAGAAAATAGGG & $61.5^{\circ} \mathrm{C}$ & 113 \\
\hline
\end{tabular}




\begin{tabular}{|c|l|l|l|l|l|l|r|}
14 & PIWI2 & CG6122 & TCTTCATCAGGTGACCCGAGA & $62.1^{\circ} \mathrm{C}$ & CTTCACGCCTGGGAGCTTC & $62.7^{\circ} \mathrm{C}$ & 85 \\
\hline 15 & Prohibitin & CG10691 & AGCCGCCTATGGAGTCAGT & $62.4^{\circ} \mathrm{C}$ & CTCGGAGTAAATGTCGCTCTG & $60.2^{\circ} \mathrm{C}$ & 103 \\
\hline 16 & R2D2 & CG7138 & TGATGAAGGATTCGACTGTGGG & $61.7^{\circ} \mathrm{C}$ & GGACGCAGTAGTCACGCAG & $62.7^{\circ} \mathrm{C}$ & 90 \\
\hline 17 & Relish & CG11992 & TGGATACCATCAAAATGGCCTG & $60.4^{\circ} \mathrm{C}$ & CTTGTACCGAAAGCGGAACTT & $60.6^{\circ} \mathrm{C}$ & 103 \\
\hline 18 & RNBP21 & CG12234 & TTAAGGAGGAGTCGCCAATGT & $60.9^{\circ} \mathrm{C}$ & TCGCACTTGCTGGTTAGACTG & $62.3^{\circ} \mathrm{C}$ & 77 \\
\hline 19 & STAT & CG4257 & CCTCGGTATGGTCACACCC & $61.4^{\circ} \mathrm{C}$ & TGCCAAACTCATTGAGGGACT & $61.4^{\circ} \mathrm{C}$ & 77 \\
\hline 20 & Toll & CG5490 & AATCCCACGTTTAGGCTAACCA & $61.4^{\circ} \mathrm{C}$ & CCTCACCGATCCGCAACTT & $62^{\circ} \mathrm{C}$ & 112 \\
\hline 21 & VIG1 & CG4170 & ATGGACAGCGCCGGTAAAAAT & $62.7^{\circ} \mathrm{C}$ & GCTGACGGTTGCTTCTTCTTG & $61.8^{\circ} \mathrm{C}$ & 134 \\
\hline
\end{tabular}


Table 2: Molecular identification of DENV-2 binding proteins from $D$. melanogaster midgut.

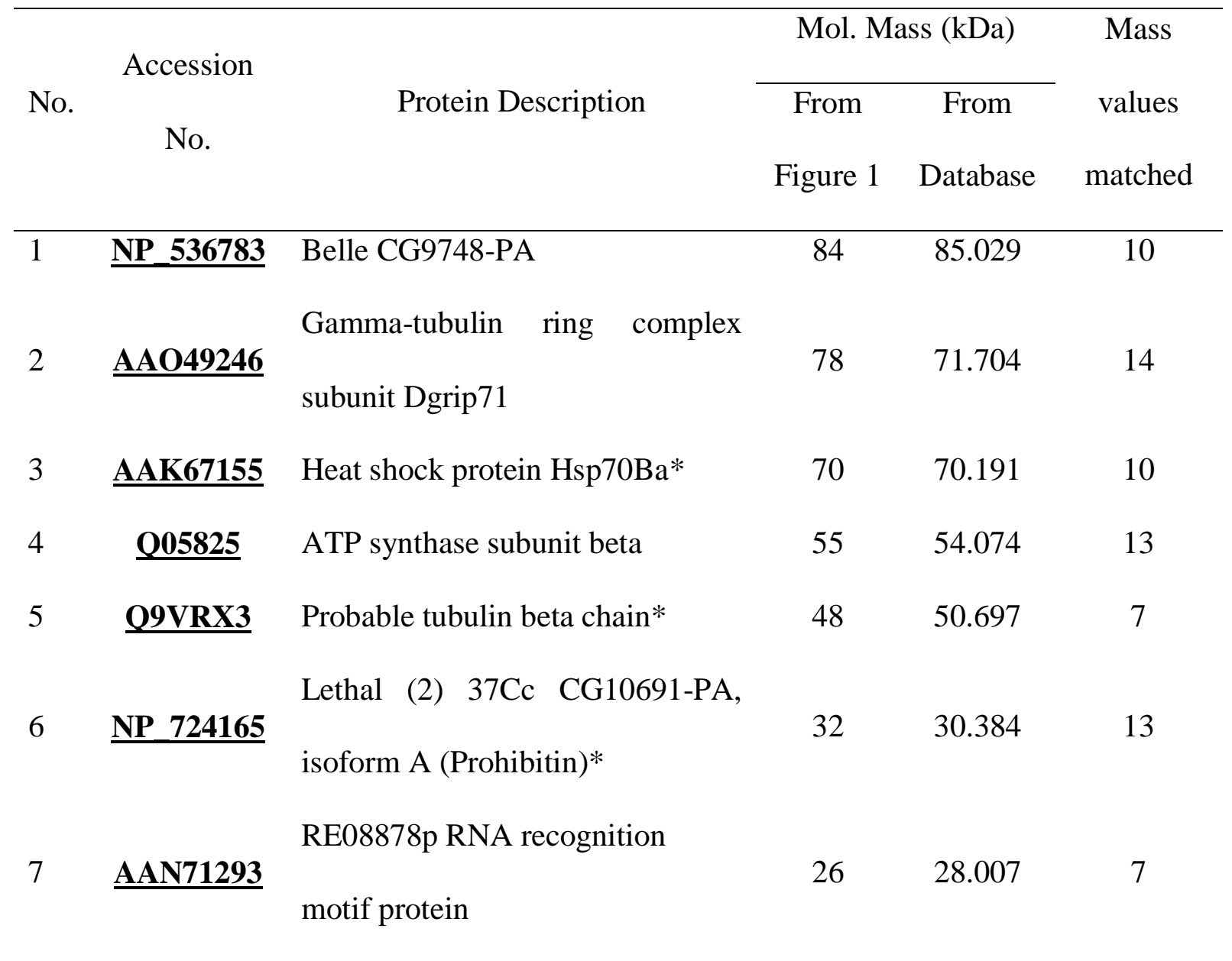

Sequence coverage is over $20 \%$ in all samples. One dimensional VOPBA and two dimensional VOPBA showed similar results therefore only results obtained in one dimensional VOPBA are listed in Table 1. Additional information on protein identification from one dimensional and two dimensional VOPBA is given in Supplementary Information Table S2.

* The monoclonal anti-prohibitin antibody (Abcam, USA) interacted with 32-kDa protein, antitubulin antibody (Sigma Aldrich, Germany) recognized $48 \mathrm{kDa}$ protein and anti-HSP70 antibody (Abcam, USA) recognized the $70-\mathrm{kDa}$ protein in brush border membrane fractions of $D$. melanogaster midgut. 
Table 3: Relative gene expression changes in D. melanogaster in response to DENV-2 infection

\begin{tabular}{|c|c|c|c|}
\hline \multirow[b]{2}{*}{ Gene Name } & \multicolumn{3}{|c|}{ Fold change mean (SD) } \\
\hline & 24 hours p.i. & 48 hours p.i. & 7 days p.i. \\
\hline \multicolumn{4}{|c|}{ miRNAi pathway } \\
\hline Argonaute 1 & $1.868(0.636)$ & $1.351(0.221)$ & $2.883(0.521)^{*}$ \\
\hline DICER 1 & $1.828(0.344)$ & $2.698(0.413) *$ & $3.247(0.433) *$ \\
\hline Drosha & $1.109(0.282)$ & $0.827(0.005)$ & $2.168(0.101) *$ \\
\hline Pasha & $0.979(0.170)$ & $0.774(0.061)$ & $2.738(0.002) *$ \\
\hline ADAR & $0.619(0.164)$ & $0.425(0.020) *$ & $0.441(0.016) *$ \\
\hline RNBP21 & $1.015(0.253)$ & $0.844(0.024)$ & $1.702(0.199)$ \\
\hline \multicolumn{4}{|c|}{ piRNAi pathway } \\
\hline Argonaute 3 & $1.036(0.318)$ & $1.067(0.177)$ & $3.216(0.613) *$ \\
\hline AUB & $1.242(0.218)$ & $1.328(0.184)$ & $2.808(0.229) *$ \\
\hline PIWI & $0.760(0.237)$ & $0.889(0.130)$ & $1.989(0.076) *$ \\
\hline \multicolumn{4}{|c|}{ siRNAi pathway } \\
\hline Argonaute 2 & $2.806(1.258)$ & $2.435(0.138) *$ & $2.415(0.537) *$ \\
\hline DICER 2 & $1.063(0.282)$ & $0.829(0.052)$ & $1.982(1.706) *$ \\
\hline R2D2 & $1.095(0.283)$ & $0.84(0.088) 2$ & $2.444(0.076) *$ \\
\hline Loqs & $0.579(0.049)$ & $0.399(0.044)$ & $0.508(0.095)$ \\
\hline FMR & $0.955(0.288)$ & $0.742(0.087)$ & $2.749(0.171) *$ \\
\hline VIG & $1.071(0.329)$ & $0.602(0.160)$ & $0.744(0.008)$ \\
\hline \multicolumn{4}{|c|}{ Other immune related genes } \\
\hline JAK & $4.498(1.061) *$ & $4.030(0.734) *$ & $4.655(1.207)$ \\
\hline STAT & $1.695(0.659)$ & $2.963(0.653) *$ & $3.211(0.893) *$ \\
\hline Prohibitin & $1.177(0.271)$ & $1.292(0.465)$ & $1.723(0.228) *$ \\
\hline Rel1 & $1.421(0.336)$ & $1.010(0.106)$ & $1.760(0.134)$ \\
\hline Toll & $1.866(0.438)$ & $2.576(1.072)$ & $4.131(0.370) *$ \\
\hline
\end{tabular}

The quantitative expression of the target gene was normalized to $18 \mathrm{~s}$ mRNA in the same samples. * Significant difference (Mann-Whitney U test $P<0.05$ ). 
A

Heat shock protein $\mathrm{Hsp} 70 \mathrm{Ba}$ ATP synthase subunit beta Probable tubulin beta chain

Belle CG9748-PA Dgrip71

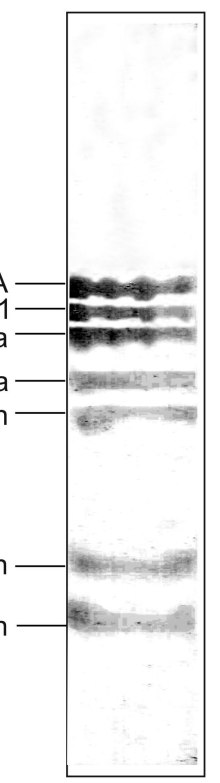

L1

B

Re08878p RNA recognition motif protein

Prohibitin 
A

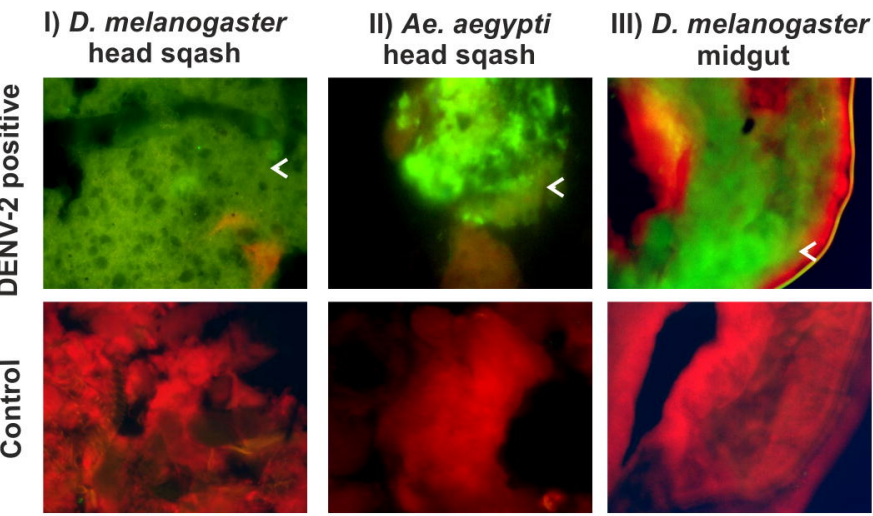

C

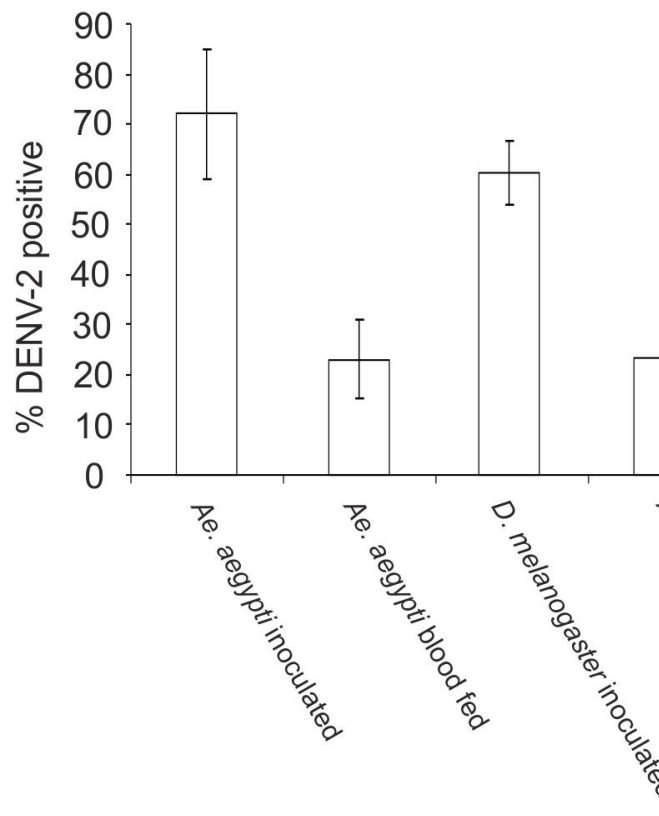

DENV-2
B

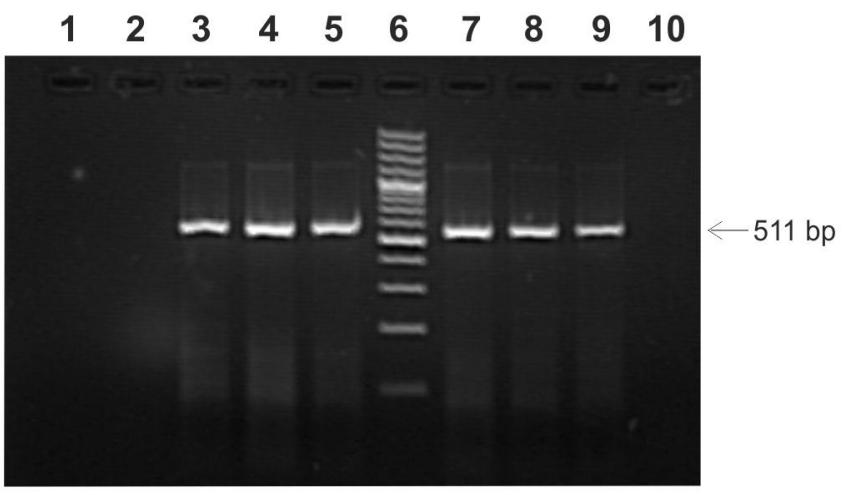

D
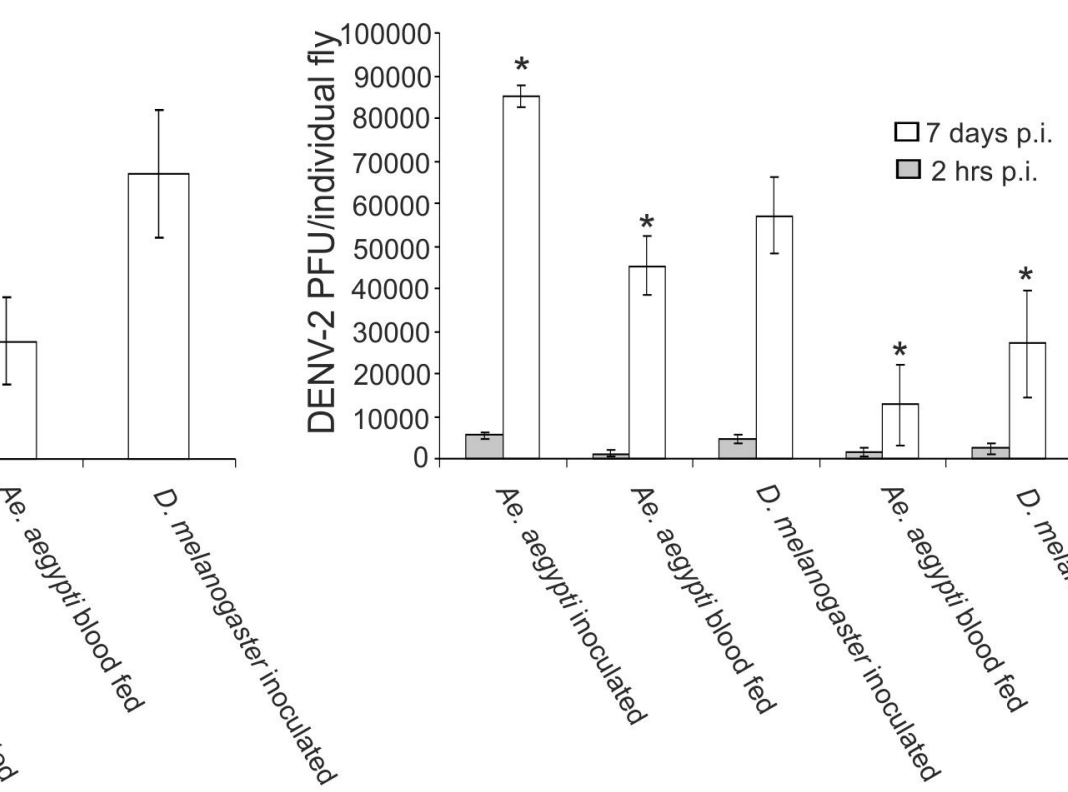

D. melanogaster generated DENV-2 $\square 2$ hrs p.i.

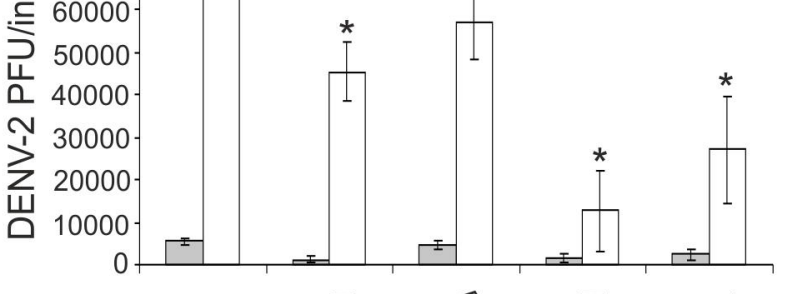

布

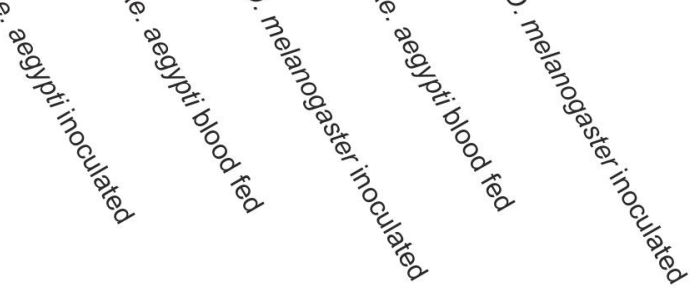

DENV-2
D. melanogaster generated DENV-2 